\title{
Magnetic phase diagram of the $S=1 / 2$ antiferromagnetic zigzag spin chain in the strongly frustrated region: cusp and plateau
}

\author{
Kouichi Okunishi and Takashi Tonegawa ${ }^{1}$ \\ Department of Physics, Faculty of Science, Niigata University, Igarashi 2, 950-2181, Japan \\ ${ }^{1}$ Department of Mechanical Engineering, Fukui University of Technology, 6-1 Gakuen 3-Chome, Fukui-Shi 910-8505, Japan.
}

(Received )

We determine the magnetic phase diagram of the $S=1 / 2$ antiferromagnetic zigzag spin chain in the strongly frustrated region, using the density matrix renormalization group method. We find the magnetization plateau at $1 / 3$ of the full moment accompanying the spontaneous symmetry breaking of the translation, the cusp singularities above and/or below the plateau, and the even-odd effect in the magnetization curve. We also discuss the formation mechanisms of the plateau and cusps briefly.

KEYWORDS: frustration, cusp, $1 / 3$ plateau, even-odd effect

For the purpose of clarifying the role of the frustration in low-dimensional quantum spin systems, the $S=1 / 2$ antiferromagnetic zigzag spin chain has been attracting considerable attention, since it minimally contains the frustrating interaction without loss of the translational invariance. ${ }^{1-4)}$ The Hamiltonian of the model is given by

$$
\mathcal{H}=\sum_{i}\left[J_{1} \vec{S}_{i} \cdot \vec{S}_{i+1}+J_{2} \vec{S}_{i} \cdot \vec{S}_{i+2}\right]-H \sum_{i} S_{i}^{z},(1)
$$

where $\vec{S}$ is the $S=1 / 2$ spin operator, $H$ is the magnetic field, and $J_{1}$ and $J_{2}$ denote the nearest and next nearest neighbor couplings, respectively. We introduce the notation $\alpha=J_{2} / J_{1}$ for simplicity. Recently, the zigzag chain was realized as $\mathrm{SrCuO}_{2},{ }^{5)} \mathrm{Cu}\left(\right.$ ampy) $\left.\mathrm{Br}_{2},{ }^{6}\right)$ $\left(\mathrm{N}_{2} \mathrm{H}_{5}\right) \mathrm{CuCl}_{3}{ }^{7)}$ and $\mathrm{F}_{2}$ PIMNH. $\left.{ }^{8}\right)$

The Hamiltonian (1) has a very simple form, but captures a variety of behaviors induced by the frustration. In particular, the zigzag chain in a magnetic field has been studied actively ${ }^{9-13)}$ and it has been clarified that cusp singularities appear near the saturation field and/or in the low field region for $\alpha \leq 0.6$, in accordance with the frustration-driven shape change of the dispersion curve of the elementary excitations. ${ }^{12-14)}$ However, the magnetic phase diagram including the strongly frustrated region $(\alpha>0.6)$ has not been acquired yet. Here we remark that the magnetization curve of $\alpha=0.6$ is still quite different from the one in the $\alpha \rightarrow \infty$ limit (see Fig.2 (a)). Thus, as $\alpha$ is increased beyond $\alpha=0.6$, the intrinsic structural change of the magnetization curve can be expected particularly around $\alpha \simeq 1$, where the most significant competition is achieved.

In this paper we address the magnetization process of the zigzag chain in the strongly frustrated region $(\alpha>$ 0.6 ), using the density matrix renormalization group (DMRG) method. ${ }^{15)}$ We then find that the obtained phase diagram exhibits rich physics, as is shown in Fig.1. For $0.56 \lesssim \alpha \lesssim 1.25$, the magnetization plateau appears at $1 / 3$ of the full moment, accompanying the spontaneous breaking of the translational symmetry with the period three. Moreover, the cusp singularities in the magnetization curve show quite interesting behavior; as $\alpha$ is increased, the high field cusp merges into the $1 / 3$ plateau at $\alpha \simeq 0.82$. Also the low field cusp merges the $1 / 3$ plateau at $\alpha \simeq 0.7$, but it appears again when $\alpha>0.7$. In addition, we find an interesting even-odd effect in the magnetization curve for $\alpha>0.7$.

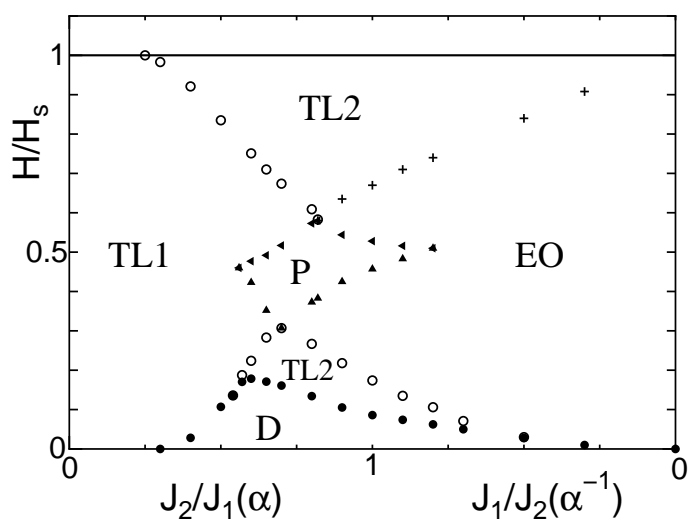

Fig. 1. Magnetic phase diagram of the zigzag spin chain. D: dimer gapped phase, P:1/3 plateau, TL1: one component TL liquid, TL2: two component TL liquid, and EO: even-odd behavior branch. The open circles denote the position of the cusp singularities. The solid circles represent the boundary of the dimer gapped phase. The triangles represent the upper and lower edges of the $1 / 3$ plateau, and the crosses indicate the upper edge of the even-odd effect in the magnetization curve. The saturation field is normalized to be unity.

In analyzing the phase diagram, an important feature of the zigzag chain is that the Hamiltonian (1) interpolates between the single Heisenberg $\operatorname{chain}\left(J_{2}=0\right)$ and the double Heisenberg one $\left(J_{1}=0\right)$ continuously. In other words, we can describe the frustration effect in the zigzag chain as the interplay between the single and double chain natures. Although such a view point has not been emphasized so far, it provides some essential insights on the frustration effect in the magnetization curve. In fact, the cusp transition for $\alpha<0.7$ mentioned above is described as the transition between the one and two component Tomonaga-Luttinger(TL) liquid; ${ }^{12)}$ the two component TL liquid is realized below the lower cusp and/or above the upper cusp reflecting the two chain nature of the system, while the middle-field branch still 
consists of the one component TL liquid. In the following, we show the results of the magnetization curves for $\alpha>0.6$ and then proceed to a detailed analysis of each phase. How can we connect the above noted competition with the characteristic behaviors in each phase?

magnetization curves: In order to compute the magnetization curve, we employ the DMRG of both the finite system size and infinite system size versions. However, here we mainly present the results for the finite size systems. We compute the minimum energy $E_{N}(M)$ for a magnetization $M$ of the $N=192$ site system with the free boundary condition, and then obtain the magnetization curve by determining the level crossing point from $E_{N}(M)$ and $E_{N}(M \pm 1)$. The maximum number of the retained bases in the DMRG computation is typically 60 , where the energy is converged sufficiently.

In Fig.2 (a) we show the magnetization curve for $\alpha=0.6$, where the scale of the magnetic field is normalized with the saturation field $H_{s}$. The cusp singularities at $H / H_{s} \simeq 0.22$ and $H / H_{s} \simeq 0.75$ are the same as those reported in the previous papers, which are explained well by the shape change of the spinon or spin wave dispersion curves. ${ }^{13,14)}$ A novel point in the figure is that the $1 / 3$ plateau is found to appear accompanying the spontaneous symmetry breaking of the translational invariance. The lower critical field of this plateau is $H_{c 1} / H_{s}=0.424$ and the upper critical field is $H_{c 2} / H_{s}=0.477$. The details of the $1 / 3$ plateau are discussed later.

As $\alpha$ is increased, the cusps approach the plateau, which implies that the two component TL region becomes more dominant, and at the same time the width of the plateau extends. Figure 2 (b) shows the magnetization curve of $\alpha=0.7$, in which the width of the $1 / 3$ plateau is extended to 0.209 . An anomalous step corresponding to $M=33$ in the $1 / 3$ plateau is due to the open boundary effect. By analyzing the size dependence, we have confirmed that this step is almost independent of $N$ and is thus negligible in the bulk limit. In addition to the plateau, an interesting point is that the low field cusp is merged into the $1 / 3$ plateau, and then the branch below the plateau sticks into the plateau with infinite slope. On the other hand, the high field cusp still survives.

In Fig.2 (c), we show the result for $\alpha=0.8$. In the figure we can clearly see that the high field cusp almost merges into the $1 / 3$ plateau; a precise computation indicates that the disappearing point of the high field cusp is $\alpha \simeq 0.82$. On the other hand, a cusp singularity appears again in the low field region and, moreover, the magnetization curve above this low field cusp remarkably exhibits an anomalous even-odd oscillation with respect to the magnetization $M$. Of course, the anomalous steps corresponding to $M=$ odd must be skipped in the true magnetization curve of the finite size system which is determined from $E_{N}(M)$ with $M=$ even. ${ }^{16)}$ However, we purposely show these anomalous odd steps, since they are reflecting an interesting aspect of the double-chains nature of the system. We discuss the details of this evenodd effect later, but here simply note that the magnetization curve obtained with the infinite system DMRG is consistent with the present 192-site result.
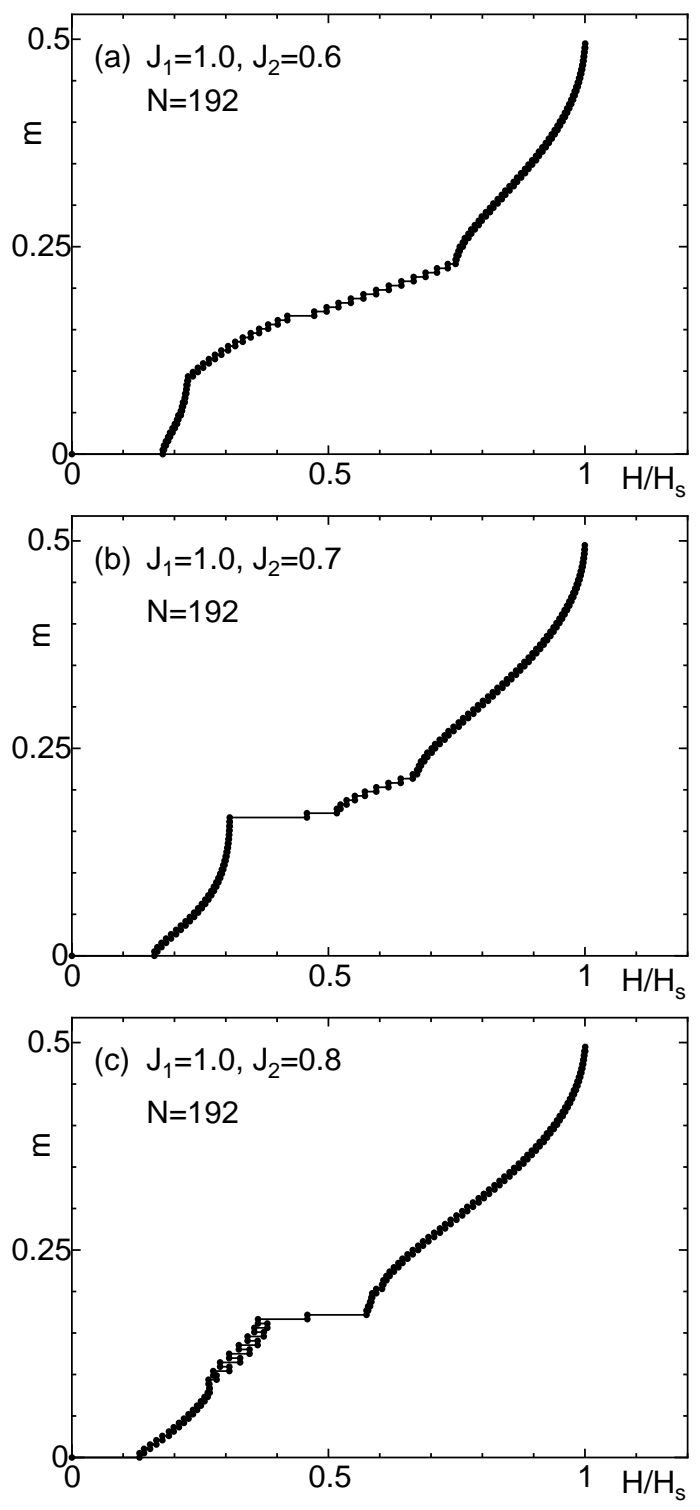

Fig. 2. Magnetization curves for $\alpha=0.6(\mathrm{a}), 0.7(\mathrm{~b})$ and $0.8(\mathrm{c})$. The vertical axis indicates $m \equiv M / N$.

As $\alpha$ is increased beyond $\alpha=0.82$, the $1 / 3$ plateau turns to shrink. We show the results for $\alpha=1.0$ in Fig.3 (a), where the plateau becomes narrower compared with that for the $\alpha=0.8$ result and the cusp shifts to the lower field side. However, the high field cusp does not appear again. Instead, the even-odd behavior of the magnetization curve is extended above the $1 / 3$ plateau. This upper edge of the even-odd effect shifts to the higher field side as $\alpha$ is increased. Although there is a possibility of the cusp appearing at the upper edge of the evenodd branch, we can not confirm it within the present numerical results for 192 sites.

Figure 3(b) shows the magnetization curve of $\alpha^{-1}=$ 0.8. In the figure, the region of the even-odd oscillation is extended to a higher field region and the plateau becomes indistinct. Indeed, for $\alpha^{-1}=0.8$, the infinite system DMRG illustrates a continuous curve above the low filed cusp up to the saturation field, implying that there is no $1 / 3$ plateau. As $\alpha$ is increased further, the branch above the cusp becomes more similar to that of 

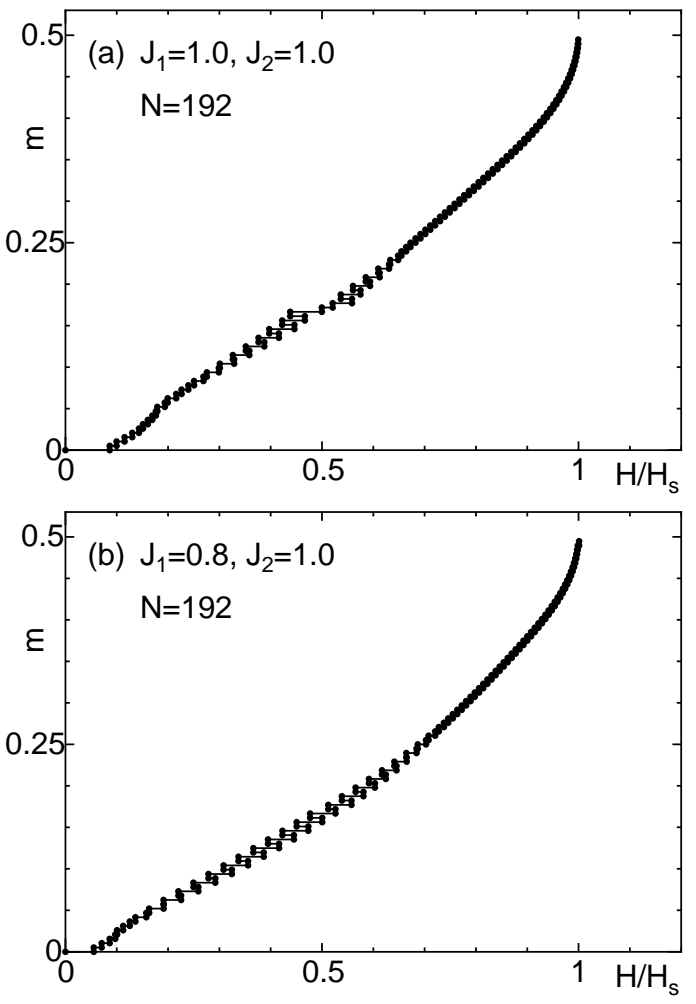

Fig. 3. Magnetization curves for $\alpha=1.0$ (a) and $\alpha^{-1}=0.8(\mathrm{~b})$.

the (double) Heisenberg chain. At the same time, the low field cusp itself approaches the dimer gap boundary. In the $J_{1} \rightarrow 0\left(\alpha^{-1} \rightarrow 0\right)$ limit, the "amplitude" of the even-odd oscillation becomes smaller and finally the magnetization curve becomes equivalent to that of the Heisenberg chain again. However, we note that it is difficult to see how the cusp behaves in this limit simply by the direct observation of the magnetization curve.

$1 / 3$ plateau state: Let us discuss the nature of the $1 / 3$ plateau. According to the quantization condition of the magnetization plateau, ${ }^{17)}$ the $1 / 3$ plateau state satisfies $q(1 / 2-M)=$ integer with $M=1 / 6$, where $q$ is the period of the plateau state. This implies that the translational symmetry of the Hamiltonian (1) must be spontaneously broken with $q=3,6, \cdots$. In order to see it, we calculate the local spin moments at the plateau along the chain direction. The result is shown in Fig.4, where the value of the local moment is developed to about $\left\langle S_{i}^{z}\right\rangle \simeq 0.38$ or -0.26 and thus the "up-up-down" type long-range order is realized. Moreover, the boundary effect decays rapidly, which supports that the $1 / 3$ plateau state has the excitation gap.

What is the origin of this up-up-down structure? We have also investigated the magnetization curve of the zigzag XXZ chain with the Ising-like anisotropy, and then found that the $1 / 3$ plateau is extended up to the Ising limit. Here it should be remarked that the $1 / 3$ plateau of the up-up-down array is proven for the Ising anisotropic limit, ${ }^{18)}$ where the up-up-down structure is especially compatible with the triangle structure of the zigzag chain. In addition, we have also checked that a similar $1 / 3$ plateau of the up-up-down array appears for the Ising-like classical XXZ zigzag chain reflecting the triangular structure. ${ }^{19)}$ However, this "classical" plateau vanishes at the isotropic XXX chain. Thus the present $1 / 3$ plateau is induced by the quantum effect.

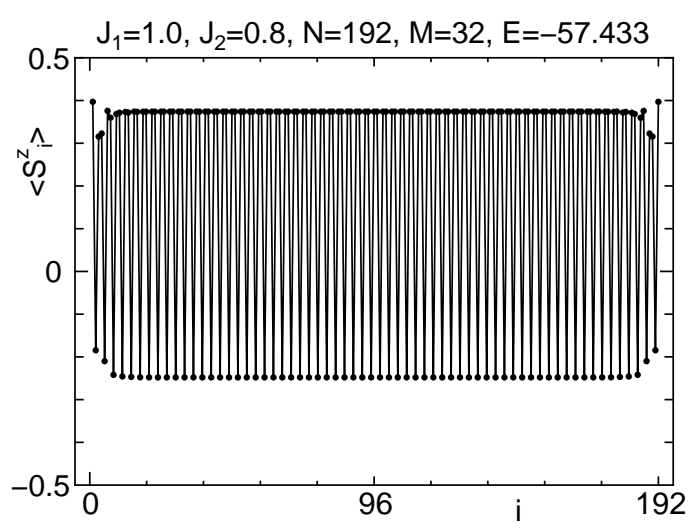

Fig. 4. The distribution of the local spin moment at $M=32(1 / 3$ plateau) for $\alpha=0.8$

even-odd effect: The anomalous even-odd behavior for $\alpha>0.7$ can be explained in terms of the formation of the bound state of magnetic quasi particles as follows. The spin chain with a finite magnetization is described by the quasi particles carrying $S^{z}=1$ distributed on the chain. ${ }^{20}$ ) When $\alpha$ is small and the single chain nature is dominant, the magnetic particle is distributed on the single chain, but if $\alpha$ becomes large and the double chain nature becomes significant, the particles should be distributed on each chain equivalently. However, if the total magnetization is odd, the remaining one particle is sitting on one of the two chains. Thus the $M=$ odd system is less stable than the $M=$ even one, which suggests the formation of the bound state in the thermodynamic limit. In order to see it, we define the binding energy of the $N$-site system as $\Delta_{N}(m) \equiv 2\left(E_{N}(M+1)-E_{N}(M)\right)-\left(E_{N}(M+\right.$ $\left.2)-E_{N}(M)\right)=2 E_{N}(M+1)-E_{N}(M)-E_{N}(M+2)$ for $M=$ even. Figure 5 shows the obtained result with the size extrapolation of $\Delta_{N}(m) \sim \Delta(m)+$ const $/ N$ for $N=240,192,168,120,96,84,{ }^{21)}$ which shows clearly that the binding energy develops in the region corresponding to the even-odd behavior. Here we note that this size extrapolation does not work well around the low field $\operatorname{cusp}(m \simeq 0.6)$ due to the singular behavior.

The formation of the bound state of the quasi particles provides an essential insight into the cusp mechanism for $\alpha>0.7$. The magnetization curve below the low field cusp consists of the two-component TL liquid that is continuously connected from the $\alpha<0.7$ region, while the branch above the low field cusp consists of the bound state of the magnetic particles. Thus the cusp mechanism for $\alpha>0.7$ is described by the transition from the two component TL liquid to the single component TL liquid consisting of the bound state, which is completely different from the mechanism for $\alpha<0.7$ based on the shape change of the dispersion curve of the spinon excitation carrying $S^{z}=1 / 2$.

To summarize, we have presented the magnetic phase diagram of the $S=1 / 2$ zigzag spin chain including the strongly frustrated region, based on the 192-site results. 


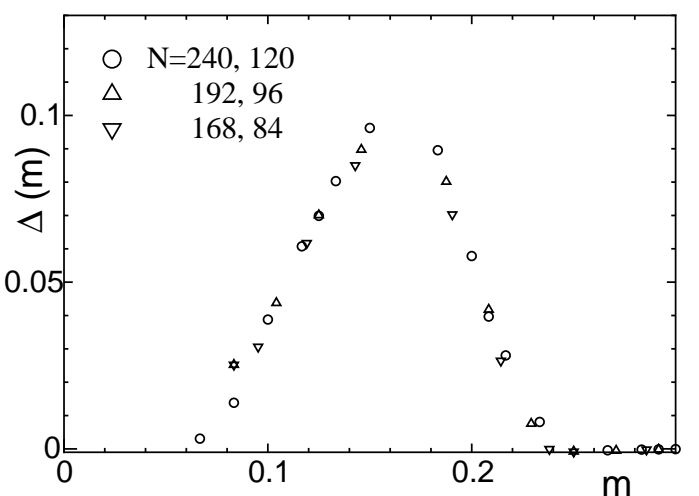

Fig. 5. The binding energy for $\alpha=1.0$ obtained by the size extrapolation: $\Delta_{N}(m) \sim \Delta(m)+$ const $/ N$. The open circles denote the result for $N=240$ and 120 . The upward and downward triangles denote those for $N=192$ and 96 , and 168 and 84 respectively. These results are in good agreements with each other.

Although we can not eliminate the possibility of a "fine structure" appearing in the magnetization curve beyond the resolution of the present calculation, we believe that the phase boundaries of the cusps and plateau are correctly obtained. The resulting phase diagram in Fig.1 exhibits a variety of interesting physics: The $1 / 3$ plateau accompanying the spontaneous breaking of the translational symmetry, the characteristic move of the cusp singularities around the plateau and the even-odd behavior of the magnetization curve. In particular we have clarified two types of cusp mechanisms: the cusp based on the shape change of the dispersion curve and the cusp attributed to the formation of the bound state related to the even-odd behavior of the magnetization curve. In the background of these behaviors, we have focused on the role of the frustration in terms of the competition between the single and double chain properties. The magnetic field triggers the switching of these two natures which induces the cusp transitions. In addition, the $1 / 3$ plateau and the cusps remarkably merge with each other at $\alpha \simeq 0.7$ and 0.82 . However, the details of the mechanism for this remain an interesting future problem.

Although the zigzag chain is a quite simple model, we consider that it captures the intrinsic aspects common to a class of frustrated spin chains having the zigzag type structure. For the exploration of such models, we believe that our result is instructive and useful.

We would like to thank T. Hikihara for valuable comments. K.O. also thanks A. Koga and A. Kawaguchi for fruitful discussions. This work is partially supported by a Grant-in-Aid for Scientific Research on Priority Areas (B) from the Ministry of Education, Culture, Sports, Science and Technology of Japan.

1) F. D. M. Haldane: Phys. Rev. B 25 (1982) 4925

2) T. Tonegawa and I. Harada: J. Phys. Soc. Jpn. 56 (1987)2153;

3) K. Okamoto and K. Nomura: Phys. Lett. A 169 (1992) 433;

4) S. R. White and I. Affleck: Phys. Rev. B 54 (1996) 9862.

5) M. Matsuda and K. Katsumata: J. Mag. Mag. Mat. 140144,(1995) 1671.

6) H. Kikuchi, H. Nagasawa, Y. Ajiro, T. Asano, and T. Goto: Physica B 284-288 (2000) 1631.

7) M. Hagiwara, Y. Narumi, K. Kindo, N. Maeshima, K. Okun-

ishi, T. Sakai, and M. Takahashi: Physica B 294-295 (2001) 83; N. Maeshima, M. Hagiwara, Y. Narumi, K. Kindo, T.C. Kobayashi and K. Okunishi: cond-mat/0208373.

8) Y. Hosokoshi, K. Katoh, K. Inoue, and T. Goto: J. Phys. Soc. Jpn. 68,(1999) 2910.

9) T. Tonegawa and I. Harada: Physica B 155 (1989) 379.

10) M. Schmidt, C. Gerhardt, K.-H. Mütter and M. Karbach: J. Phys. Condens. Matter. 8 (1996) 553.; C. Gerhard, A. Fledderjohann, E. Aysal, K-H. Mütter, J.F. Audet and H. Kröger: ibid 9 (1997) 3435.

11) D.C. Cabra, A. Honecker, and P. Pujol: Eur. Phys. J. B 13 (2000) 55.

12) K. Okunishi, Y. Hieida and Y. Akutsu: Phys. Rev. B 60 (1999) R6953.

13) N. Maeshima and K. Okunishi: Phys. Rev. B 62 (2000) 934.

14) K. Okunishi and N. Maeshima: Phys. Rev. B 64, (2001) 212406.

15) S.R. White: Phys. Rev. Lett. 692863 (1992); Phys. Rev. B 48 (1993) 10345

16) Thus the true magnetization curve passes almost through the middle of the oscillating steps.

17) M. Oshikawa, M. Yamanaka and I. Affleck: Phys. Rev. Lett. 78 (1997) 1984.

18) T. Morita and T. Horiguchi: Phys. Lett. A 38 (1972) 223.

19) We have investigated the classical chain with the conjugate gradient method. The plateau mechanism for the classical model is essentially the same as the one in S. Miyasihta: J.Phys. Soc. Jpn. 55 (1986) 3605.

20) This argument of the quasi-particle of $S^{z}=1$ is applicable to a large but finite size system.

21) The even-odd behavior branch of the magnetization curve is expected to be described by the TL liquid made from the bound state particles. Thus we can naturally assume the $1 / N$ dependence for $\Delta_{N}(m)$. 